Proyecciones Journal of Mathematics

Vol. 35, No 3, pp. 263-275, September 2016.

Universidad Católica del Norte

Antofagasta - Chile

\title{
Asymptotic stability in delay nonlinear fractional differential equations
}

\author{
A. Ardjouni \\ Univ Souk Ahras, Algeria \\ H. Boulares \\ University of Annaba, Algeria \\ and \\ A. Djoudi \\ University of Annaba, Algeria \\ Received : November 2015. Accepted : June 2016
}

\begin{abstract}
In this paper, we give sufficient conditions to guarantee the asymptotic stability of the zero solution to a kind of delay nonlinear fractional differential equations of order $\alpha(1<\alpha<2)$. By using the Banach's contraction mapping principle in a weighted Banach space, we establish new results on the asymptotic stability of the zero solution provided that $g(t, 0)=f(t, 0,0)=0$, which include and improve some related results in the literature.
\end{abstract}

Subjclass [2000] : 34K20, 34K30, 34K40.

Keywords : Delay fractional differential equations, Fixed point theory, Asymptotic stability. 


\section{Introduction}

Fractional differential equations with and without delay arise from a variety of applications including in various fields of science and engineering such as applied sciences, practical problems concerning mechanics, the engineering technique fields, economy, control systems, physics, chemistry, biology, medicine, atomic energy, information theory, harmonic oscillator, nonlinear oscillations, conservative systems, stability and instability of geodesic on Riemannian manifolds, dynamics in Hamiltonian systems, etc. In particular, problems concerning qualitative analysis of linear and nonlinear fractional differential equations with and without delay have received the attention of many authors, see [1]-[13], [15] and the references therein.

Recently, Agarwal, Zhou and He [2] discussed the existence of solutions for the neutral fractional differential equation with bounded delay

$$
\left\{\begin{array}{l}
{ }^{C} D^{\alpha}\left(x(t)-g\left(t, x_{t}\right)\right)=f\left(t, x_{t}\right), t \geq t_{0}, \\
x_{t_{0}}=\phi
\end{array}\right.
$$

where ${ }^{C} D^{\alpha}$ is the standard Caputo's fractional derivative of order $0<$ $\alpha<1$. By employing the Krasnoselskii's fixed point theorem, the authors obtained existence results.

The delay fractional differential equation

$$
\left\{\begin{array}{l}
\frac{d^{\alpha}}{d t^{\alpha}} x(t)=f(t, x(t), x(t-\tau)), t \in[0, T] \\
x(t)=\phi(t), t \in[-\tau, 0], 0<\alpha<1
\end{array}\right.
$$

has been investigated in [1], where $\frac{d^{\alpha}}{d t^{\alpha}}$ denotes Riemann-Liouville fractional derivative of order $0<\alpha<1$. By using the Krasnoselskii's fixed point theorem, the existence of solutions has been established.

In [6], Ge and Kou investigated the asymptotic stability of the zero solution of the following nonlinear fractional differential equation

$$
\left\{\begin{array}{l}
{ }^{C} D_{0+}^{\alpha} x(t)=k x(t)+f(t, x(t)), t \geq 0 \\
x^{\prime}(0)=0, x(0)=x_{0}
\end{array}\right.
$$

where ${ }^{C} D_{0+}^{\alpha}$ is the standard Caputo's fractional derivative of order $1<\alpha<$ 2. By employing the Banach's contraction mapping principle in a weighted Banach space, the authors obtained asymptotic stability results. 
In this paper, we are interested in the analysis of qualitative theory of the problems of the asymptotic stability of the zero solution to delay fractional differential equations. Inspired and motivated by the works mentioned above and the papers [1]-[13], [15] and the references therein, we concentrate on the asymptotic stability of the zero solution for the nonlinear fractional differential equation with variable delay

$\left\{\begin{array}{l}{ }^{C} D_{0+}^{\alpha} x(t)=k x(t)+f(t, x(t), x(t-\tau(t)))+{ }^{C} D_{0+}^{\alpha-1} g(t, x(t-\tau(t))), t \geq 0, \\ x^{\prime}(0)=0, x(t)=\phi(t), t \in\left[m_{0}, 0\right]\end{array}\right.$ $(1.1)$

where $1<\alpha<2, k \in \mathbf{R}$ is a given constant, $\mathbf{R}^{+}=[0,+\infty)$,

$\tau: \mathbf{R}^{+} \rightarrow \mathbf{R}^{+}$is continuous with $t-\tau(t) \rightarrow \infty$ as $t \rightarrow \infty, m_{0}=$ $\inf _{t \geq 0}\{t-\tau(t)\}, g: \mathbf{R}^{+} \times \mathbf{R} \rightarrow \mathbf{R}$ and

$f: \mathbf{R}^{+} \times \mathbf{R} \times \mathbf{R} \rightarrow \mathbf{R}$ are continuous functions and $g(t, 0)=f(t, 0,0)=$ $0,{ }^{C} D_{0+}^{\alpha}$ is the standard Caputo fractional derivative and we denote the solution of $(1.1)$ by $x(t, \phi, 0)$. To show the asymptotic stability of the zero solution, we transform (1.1) into an integral equation and then use Banach's contraction mapping principle [14].

This paper is organized as follows. In section 2, we introduce some notations and lemmas, and state some preliminaries results needed in later sections. Also, we present the inversion of (1.1). In Section 3, we give and prove our main results on stability.

\section{Preliminaries}

We introduce some necessary definitions, lemmas and theorems which will be used in this paper. For more details, see [7, 8, 13, 14].

Definition $2.1([7,13])$. The fractional integral of order $\alpha>0$ of a function $x: \mathbf{R}^{+} \rightarrow \mathbf{R}$ is given by

$$
I_{0+}^{\alpha} x(t)=\frac{1}{\Gamma(\alpha)} \int_{0}^{t}(t-s)^{\alpha-1} x(s) d s
$$

provided the right side is pointwise defined on $\mathbf{R}^{+}$. 
Definition $2.2([\mathbf{7}, \mathbf{1 3}])$. The Caputo fractional derivative of order $\alpha>0$ of a function $x: \mathbf{R}^{+} \rightarrow \mathbf{R}$ is given by

$$
{ }^{C} D_{0+}^{\alpha} x(t)=I_{0+}^{n-\alpha} x^{(n)}(t)=\frac{1}{\Gamma(n-\alpha)} \int_{0}^{t}(t-s)^{n-\alpha-1} x^{(n)}(s) d s,
$$

where $n=[\alpha]+1$, provided the right side is pointwise defined on $\mathbf{R}^{+}$.

Lemma $2.3([7,13])$. Let $\Re(\alpha)>0$. Suppose $x \in C^{n-1}[0,+\infty)$ and $x^{(n)}$ exists almost everywhere on any bounded interval of $\mathbf{R}^{+}$. Then

$$
\left(I_{0+}^{\alpha C} D_{0+}^{\alpha} x\right)(t)=x(t)-\sum_{k=0}^{n-1} \frac{x^{(k)}(0)}{k !} t^{k} .
$$

In particular, when $0<\Re(\alpha)<1,\left(I_{0+}^{\alpha}{ }^{C} D_{0+}^{\alpha} x\right)(t)=x(t)-x(0)$.

Remark 2.4. From Definitions 2.1, 2.2 and Lemma 2.3, it is easy to see that

(1) Let $\Re(\alpha)>0$. If $x$ is continuous on $\mathbf{R}^{+}$, then $D_{0+}^{\alpha} I_{0+}^{\alpha} x(t)=x(t)$ holds for all $t \in \mathbf{R}^{+}$.

(2) The Caputo derivative of a constant is equal to zero.

The following Banach space plays a fundamental role in our discussion. Let $h:\left[m_{0},+\infty\right) \rightarrow(0,1]$ be a strictly decreasing continuous function with $h\left(m_{0}\right)=1, h(t) \rightarrow 0$ as $t \rightarrow \infty$. Let

$$
E=\left\{x \in C\left(\left[m_{0},+\infty\right)\right): \sup _{t \geq m_{0}}\{h(t)|x(t)|\}<\infty\right\} .
$$

Then $E$ is a Banach space equipped with the norm $\|x\|=\sup _{t \geq m_{0}}\{h(t)|x(t)|\}$. For more properties of this Banach space, see [8]. Moreover, let

$$
\|\varphi\|_{t}=\max \left\{|\varphi(s)|: m_{0} \leq s \leq t\right\},
$$

for any $t \geq m_{0}$, any given $\varphi \in C\left(\left[m_{0},+\infty\right)\right)$ and let $\Im(\varepsilon)=\{x \in E:\|x\| \leq$ $\varepsilon$ and $x(t)=\phi(t)$ if $\left.t \in\left[m_{0}, 0\right]\right\}$ for any $\varepsilon>0$.

Lemma 2.5 ([5]). Let $r \in C\left(\left[m_{0},+\infty\right)\right)$. Then $x \in C\left(\left[m_{0},+\infty\right)\right)$ is a solution of the Cauchy type problem

$$
\left\{\begin{array}{l}
{ }^{C} D_{0+}^{\alpha} x(t)=r(t), t \in \mathbf{R}^{+}, 1<\alpha<2, \\
x^{\prime}(0)=0, x(t)=\phi(t), t \in\left[m_{0}, 0\right]
\end{array}\right.
$$


if and only if $x$ is a solution of the Cauchy type problem

$$
\left\{\begin{array}{l}
x^{\prime}(t)=I_{0+}^{\alpha-1} r(t), t \in \mathbf{R}^{+} \\
x(t)=\phi(t), t \in\left[m_{0}, 0\right]
\end{array}\right.
$$

Lemma 2.6. If $R \in C([0, \infty))$ satisfies the integral equation

$$
R(t)=1+k I_{0+}^{\alpha} R(t),
$$

then $x \in C\left(\left[m_{0},+\infty\right)\right)$ is a solution of (1.1) if and only if

$$
\begin{gathered}
x(t)=R(t) \phi(0)-g(0, \phi(-\tau(0))) \int_{0}^{t} R(s) d s \\
+\int_{0}^{t} R(t-s) g(s, x(s-\tau(s))) d s \\
+\frac{1}{\Gamma(\alpha-1)} \int_{0}^{t} R(t-s) \int_{0}^{s}(s-u)^{\alpha-2} f(u, x(u), x(u-\tau(u))) d u d s .
\end{gathered}
$$

Proof. Let $x \in C\left(\left[m_{0},+\infty\right)\right)$ be a solution of (1.1). From Lemma 2.5, we have

$$
\left\{\begin{aligned}
x^{\prime}(t) & =g(t, x(t-\tau(t)))-g(0, \phi(-\tau(0))) \\
& +\frac{k}{\Gamma(\alpha-1)} \int_{0}^{t}(t-s)^{\alpha-2} x(s) d s \\
& +\frac{1}{\Gamma(\alpha-1)} \int_{0}^{t}(t-s)^{\alpha-2} f(s, x(s), x(s-\tau(s))) d s \\
x(t) & =\phi(t), t \in\left[m_{0}, 0\right] .
\end{aligned}\right.
$$

On the other hand, for any given $t \geq 0$, similar to the argument in section 3.5 of [7], it is easy to see that there exists at least one $R \in C([0, \infty))$ satisfying (2.3). Besides, for any $R \in C([0, \infty))$ satisfying $(2.3)$, we can get that $R(0)=1, R^{\prime}(t)=k I_{0+}^{\alpha-1} R(t)$, and

$$
\frac{d R(t-s)}{d s}=\frac{-k}{\Gamma(\alpha-1)} \int_{0}^{t-s} R(t-s-v) v^{\alpha-2} d v
$$




$$
=\frac{-k}{\Gamma(\alpha-1)} \int_{s}^{t} R(t-u)(u-s)^{\alpha-2} d u \text {. }
$$

For fixed $t>0$, we can conclude that

$$
\begin{aligned}
& \int_{0}^{t}\left\{\frac{d R(t-s)}{d s} x(s)+R(t-s) x^{\prime}(s)\right\} d s \\
& =\left.R(t-s) x(s)\right|_{s=0} ^{s=t}=x(t)-R(t) \phi(0) .
\end{aligned}
$$

Considering (2.5) and (2.6), we have

$$
\begin{aligned}
& x(t)-R(t) \phi(0) \\
& =\int_{0}^{t} \frac{d R(t-s)}{d s} x(s) d s+\int_{0}^{t} \frac{k}{\Gamma(\alpha-1)} \int_{0}^{s}(s-u)^{\alpha-2} x(u) d u d s \\
& +\int_{0}^{t} R(t-s)[g(s, x(s-\tau(s)))-g(0, \phi(-\tau(0)))] d s \\
& +\frac{1}{\Gamma(\alpha-1)} \int_{0}^{t} R(t-s) \int_{0}^{s}(s-u)^{\alpha-2} f(u, x(u), x(u-\tau(u))) d u d s \\
& =\int_{0}^{t}\left[\frac{d R(t-s)}{d s}+\frac{k}{\Gamma(\alpha-1)} \int_{s}^{t} R(t-u)(u-s)^{\alpha-2} d u\right] x(s) d s \\
& -g(0, \phi(-\tau(0))) \int_{0}^{t} R(s) d s+\int_{0}^{t} R(t-s) g(s, x(s-\tau(s))) d s \\
& +\frac{1}{\Gamma(\alpha-1)} \int_{0}^{t} R(t-s) \int_{0}^{s}(s-u)^{\alpha-2} f(u, x(u), x(u-\tau(u))) d u d s \\
& =-g(0, \phi(-\tau(0))) \int_{0}^{t} R(s) d s+\int_{0}^{t} R(t-s) g(s, x(s-\tau(s))) d s \\
& +\frac{1}{\Gamma(\alpha-1)} \int_{0}^{t} R(t-s) \int_{0}^{s}(s-u)^{\alpha-2} f(u, x(u), x(u-\tau(u))) d u d s .
\end{aligned}
$$

Since each step is reversible, the converse follows easily. This completes the proof.

Definition 2.7. The trivial solution $x=0$ of (1.1) is said to be (i) stable in Banach space $E$, if for every $\varepsilon>0$, there exists a $\sigma=\sigma(\varepsilon)>0$ such that $|\phi(t)| \leq \sigma$ implies that the solution $x(t)=x(t, \phi, 0)$ exists for all $t \geq m_{0}$ and satisfies $\|x\| \leq \varepsilon$.

(ii) asymptotically stable, if it is stable in Banach space $E_{*}$ and there exists a number $\sigma>0$ such that $|\phi(t)| \leq \sigma$ implies $\lim _{t \rightarrow \infty} x(t)=0$. 


\section{Main results}

In this section, we shall present and prove our main results. First, we define the operator $T: E \rightarrow E$ as follows, $(T x)(t)=\phi(t)$ if $t \in\left[m_{0}, 0\right]$, while, for $t>0$

$$
\begin{aligned}
& (T x)(t)=R(t) \phi(0)-g(0, \phi(-\tau(0))) \int_{0}^{t} R(s) d s \\
& +\int_{0}^{t} R(t-s) g(s, x(s-\tau(s))) d s \\
& +\frac{1}{\Gamma(\alpha-1)} \int_{0}^{t} R(t-s) \int_{0}^{s}(s-u)^{\alpha-2} f(u, x(u), x(u-\tau(u))) d u d s \\
& =R(t) \phi(0)-g(0, \phi(-\tau(0))) \int_{0}^{t} R(s) d s \\
& +\int_{0}^{t}[R(t-s) g(s, x(s-\tau(s)))+K(t-s) f(s, x(s), x(s-\tau(s)))] d s
\end{aligned}
$$

where

$$
K(t)=\frac{1}{\Gamma(\alpha-1)} \int_{0}^{t}(t-u)^{\alpha-2} R(u) d u .
$$

We introduce the following hypotheses.

(h1) There exists a constant $M_{1}>0$ such that

$$
\sup _{t \geq 0} \int_{0}^{t} h(t)|R(s)| d s \leq M_{1}
$$

(h2) There exists a constant $\nu \in(0,1)$ such that

$$
\sup _{t \geq 0} \frac{|k|}{\Gamma(\alpha)} \int_{0}^{t}(t-s)^{\alpha-2} h(t) h^{-1}(s) d s=\nu .
$$

(h3) $g$ and $f$ are continuous functions and $g(t, 0)=f(t, 0,0)=0$. Also, there exist constants $\beta \in(0,1), l>0$ and continuous functions $L_{g}, L_{f 1}, L_{f 2}$ : $\mathbf{R}^{+} \rightarrow \mathbf{R}^{+}$such that

$$
\sup _{t \geq 0} \int_{0}^{t} h(t)\left\{|R(t-s)| L_{g}(s)+|K(t-s)|\left[L_{f 1}(s)+L_{f 2}(s)\right]\right\} d s \leq \beta
$$




$$
|g(t, x)-g(t, y)| \leq h(t) L_{g}(t)|x-y|
$$

and

$$
|f(t, x, y)-f(t, z, w)| \leq h(t)\left(L_{f 1}(t)|x-z|+L_{f 2}(t)|y-w|\right),
$$

for all $t \geq 0,|x|,|y|,|z|,|w| \leq l$.

Theorem 3.1. Suppose that $(h 1)-(h 3)$ hold. Then the trivial solution $x=0$ of (1.1) is stable in Banach space $E$.

Proof. Since $g$ and $f$ are continuous functions with $g(t, 0)=f(t, 0,0)=$ 0 , for each continuous functions $L_{g}, L_{f 1}$ and $L_{f 2}$ satisfying (3.2), by (3.3) and (3.4), we have

$$
|g(t, x)| \leq L_{g}(t)\|x\| \text { and }|f(t, x, y)| \leq L_{f 1}(t)\|x\|+L_{f 2}(t)\|y\|,
$$

for all $\|x\|,\|y\| \leq l$. Next, we will prove that for any $\varepsilon \in(0, l]$, there exists a $\sigma>0$ such that for $|\phi(t)| \leq \sigma$, the unique solution $x$ of (1.1) satisfies $\|x\| \leq \varepsilon$.

Let $\varepsilon>0(\varepsilon \in(0, l])$ be given. For all $t \geq 0$, we have

$$
\begin{aligned}
& h(t)|R(t)|=\left|h(t)+\frac{k}{\Gamma(\alpha)} \int_{0}^{t}(t-s)^{\alpha-1} h(t) R(s) d s\right| \\
& \leq 1+\frac{|k|}{\Gamma(\alpha)} \int_{0}^{t}(t-s)^{\alpha-1} h(t) h^{-1}(s) d s \sup _{s \geq 0}\{h(s)|R(s)|\} \\
& \leq 1+\nu \sup _{s \geq 0}\{h(s)|R(s)|\},
\end{aligned}
$$

then

$$
\sup _{s \geq 0}\{h(s)|R(s)|\} \leq \frac{1}{1-\nu}
$$

Let $M_{2}=\sup _{s \geq 0}\{h(s)|R(s)|\}$ and $\sigma=\frac{1-\beta}{M_{2}+M_{1} L_{g}(0)} \varepsilon$. Obviously, $\Im(\varepsilon) \subseteq E$ is a closed convex subset and the operator $T$ is continuous. Then for any given $x \in \Im(\varepsilon),|\phi(t)| \leq \sigma$, we have

$$
\mathrm{h}(t)|(T x)(t)|
$$

$$
\leq h(t)|R(t)||\phi(0)|+h(t)|g(0, \phi(-\tau(0)))| \int_{0}^{t}|R(s)| d s
$$




$$
\begin{aligned}
& +\mathrm{h}(t) \int_{0}^{t}[|R(t-s)||g(s, x(s-\tau(s)))|+|K(t-s)||f(s, x(s), x(s-\tau(s)))|] d s \\
& \leq M_{2}|\phi(0)|+M_{1} L_{g}(0)|\phi(-\tau(0))| \\
& +\int_{0}^{t} h(t)\left\{|R(t-s)| L_{g}(s)+|K(t-s)|\left[L_{f 1}(s)+L_{f 2}(s)\right]\right\} d s\|x\| \\
& \leq M_{2}|\phi(0)|+M_{1} L_{g}(0)|\phi(-\tau(0))|+\beta\|x\| \leq \varepsilon .
\end{aligned}
$$

Therefore, $T: \Im(\varepsilon) \rightarrow \Im(\varepsilon)$. Furthermore,

$$
\begin{aligned}
& h(t)|(T x)(t)-(T y)(t)| \\
& \leq h(t) \int_{0}^{t}[|R(t-s)||g(s, x(s-\tau(s)))-g(s, y(s-\tau(s)))| \\
& +|K(t-s)||f(s, x(s), x(s-\tau(s)))-f(s, y(s), y(s-\tau(s)))| d s \\
& \leq \int_{0}^{t} h(t)\left\{|R(t-s)| L_{g}(s)+|K(t-s)|\left[L_{f 1}(s)+L_{f 2}(s)\right]\right\} d s\|x-y\| \\
& \leq \beta\|x-y\| .
\end{aligned}
$$

Then $|(T x)(t)-(T y)(t)| \leq \beta\|x-y\|(0<\beta<1)$ is a contraction. It follows from the Banach contraction mapping principle, that $T$ has a unique fixed point $x \in \Im(\varepsilon)$, which is also the solution of (1.1).

On the other hand, for any solution $x$ of (1.1) and any $\varepsilon>0$, there exists a $\sigma=\frac{1-\beta}{M_{2}+M_{1} L_{g}(0)} \varepsilon$ such that $|\phi(t)| \leq \sigma$ implies that

$$
\begin{aligned}
& \|x\|=\sup _{t \geq m_{0}}\{h(t)|(T x)(t)|\} \\
& \leq M_{2}|\phi(0)|+M_{1} L_{g}(0)|\phi(-\tau(0))| \\
& +\sup _{t \geq 0} \int_{0}^{t} h(t)\left\{|R(t-s)| L_{g}(s)+|K(t-s)|\left[L_{f 1}(s)+L_{f 2}(s)\right]\right\} d s\|x\| \\
& \leq M_{2}|\phi(0)|+M_{1} L_{g}(0)|\phi(-\tau(0))|+\beta\|x\| \leq \varepsilon .
\end{aligned}
$$

Thus the trivial solution of (1.1) is stable in the Banach space $E$.

Before showing our main results on the asymptotic stability of (1.1), we introduce two new Banach spaces

$$
E_{*}=\left\{x \in C\left(\left[m_{0}, \infty\right)\right): \lim _{t \rightarrow \infty} x(t)=0\right\},
$$

and 


$$
E_{\beta}=\left\{x \in C\left(\left[m_{0}, \infty\right)\right): \lim _{t \rightarrow \infty} t^{\alpha-B} x(t)=0,0<\beta<1<\alpha<2\right\}
$$

equipped with the norms $\|x\|_{E_{*}}=\sup _{t \geq m_{0}}|x(t)|$ and

$\|x\|_{E_{\beta}}=\sup _{t \geq m_{0}}\left\{t^{\alpha-B}|x(t)|\right\}$, respectively. Obviously, $E_{\beta} \subseteq E_{*} \subseteq E$.

Let $\Im_{*}=\left\{x \in E_{*}: x(t)=\phi(t)\right.$ if $\left.t \in\left[m_{0}, 0\right]\right\}$.

Theorem 3.2. Suppose that the condition $(h 3)$ of Theorem 3.1 with $h(t)=$ 1 holds, $1<\alpha<1+\beta, \lim _{t \rightarrow \infty} t^{\alpha-B} R(t)=0, \lim _{t \rightarrow \infty} \int_{0}^{t} R(s) d s=0$ and

$$
\lim _{t \rightarrow \infty} \int_{0}^{t}\left\{|R(t-s)| L_{g}(s)+|K(t-s)|\left[L_{f 1}(s)+L_{f 2}(s)\right]\right\} d s=0 .
$$

Then the trivial solution of (1.1) is asymptotically stable in the Banach space $E_{*}$.

Proof. Similar to the argument in $[6,7,8]$, it is easy to prove that there exists at least one solution of (2.5) in the Banach space $E_{\beta}$. Moreover, if $\lim _{t \rightarrow \infty} t^{\alpha-B} R(t)=0$, we have $\lim _{t \rightarrow \infty} R(t)=0$ and

$$
\begin{aligned}
& |K(t)|=\left|\frac{1}{\Gamma(\alpha-1)} \int_{0}^{t}(t-u)^{\alpha-2} u^{\beta-\alpha} u^{\alpha-\beta} R(u) d u\right| \\
& \leq \frac{\Gamma(1+\beta-\alpha)}{\Gamma(\beta)} t^{\beta-1} \sup _{t \geq 0}\left\{t^{\alpha-\beta} R(t)\right\} .
\end{aligned}
$$

By Theorem 3.1, if $\lim _{t \rightarrow \infty} t^{\alpha-B} R(t)=0, \lim _{t \rightarrow \infty} \int_{0}^{t} R(s) d s=0$ and the condition $(h 3)$ of Theorem 3.1 with $h(t)=1$ holds, then the trivial solution of (1.1) is stable in the Banach space $E_{*}$. Furthermore, for any $t \geq 0$, if follows from (3.1) that

$$
\begin{aligned}
& |(T x)(t)| \leq|R(t)||\phi(0)|+L_{g}(0)|\phi(-\tau(0))|\left|\int_{0}^{t} R(s) d s\right| \\
& +\int_{0}^{t}\left\{|R(t-s)| L_{g}(s)+|K(t-s)|\left[L_{f 1}(s)+L_{f 2}(s)\right]\right\} d s\|x\|_{E_{*}} \\
& \rightarrow 0 \text { as } t \rightarrow \infty
\end{aligned}
$$

which means that $T: \Im_{*} \rightarrow \Im_{*}$. Therefore, the trivial solution of (1.1) is asymptotically stable in the Banach space $E_{*}$. 


\section{References}

[1] S. Abbas, Existence of solutions to fractional order ordinary and delay differential equations and applications, Electronic Journal of Differential Equations, Vol. 2011, No. 09, pp. 1-11, (2011).

[2] R. P. Agarwal, Y. Zhou, Y. He, Existence of fractional functional differential equations, Computers and Mathematics with Applications 59, pp. 1095-1100, (2010).

[3] T. A. Burton, B. Zhang, Fractional equations and generalizations of Schaefer's and Krasnoselskii's fixed point theorems, Nonlinear Anal. 75, pp. 6485-6495, (2012).

[4] F. Chen, J. J. Nieto, Y. Zhou, Global attractivity for nonlinear fractional differential equations, Nonlinear Analysis: Real Word Applications 13, pp. 287-298, (2012).

[5] F. Ge, C. Kou, Stability analysis by Krasnoselskii's fixed point theorem for nonlinear fractional differential equations, Applied Mathematics and Computation 257, pp. 308-316, (2015).

[6] F. Ge, C. Kou, Asymptotic stability of solutions of nonlinear fractional differential equations of order $1<\alpha<2$, Journal of Shanghai Normal University, Vol. 44, No. 3, pp. 284-290, (2015).

[7] A. A. Kilbas, H. M. Srivastava, J. J. Trujillo, Theory and Applications of Fractional Differential Equations, Elsevier, (2006).

[8] C. Kou, H. Zhou, Y. Yan, Existence of solutions of initial value problems for nonlinear fractional differential equations on the half-axis, Nonlinear Anal. 74, pp. 5975-5986, (2011).

[9] Y. Li, Y. Chen, I. Podlunby, Mittag-Leffler stability of fractional order nonlinear dynamic systems, Automatica 45, pp. 1965-1969, (2009).

[10] Y. Li, Y. Chen, I. Podlunby, Stability of fractional-order nonlinear dynamic systems: Lyapunov direct method and generalized MittagLeffler stability, Comput. Math. Appl. 59, pp. 1810-1821, (2010). 
[11] C. Li, F. Zhang, A survey on the stability of fractional differential equations, Eur. Phys. J. Special Topics. 193, pp. 27-47, (2011).

[12] I. Ndoye, M. Zasadzinski, M. Darouach, N. E. Radhy, Observerbased control for fractional-order continuous-time systems, Proceedings of the Joint 48th IEEE Conference on Decision and Control and 28th Chinese Control Conference, WeBIn5.3, pp. 1932-1937, December 1618, (2009).

[13] I. Podlubny, Fractional Differential Equations, Academic Press, San Diego, (1999).

[14] D. R. Smart, Fixed point theorems, Cambridge Uni. Press., Cambridge, (1980).

[15] J. Wang, Y. Zhou, M. Fečkan, Nonlinear impulsive problems for fractional differential equations and Ulam stability, Comput. Math. Appl. 64, pp. 3389-3405, (2012).

\section{Abdelouaheb Ardjouni}

Faculty of Sciences and Technology, Department of Mathematics and Informatics, Univ Souk Ahras, P. O. Box 1553, Souk Ahras, 41000, Algeria

e-mail: abd_ardjouni@yahoo.fr

\section{Hamid Boulares}

Depatement of Mathematics, Faculty of Sciences, University of Annaba, P. O. Box 12, Annaba, 23000, Algeria e-mail: boulareshamid@gmail.com and 


\author{
Ahcene Djoudi \\ Applied Mathematics Lab, \\ Faculty of Sciences, \\ Department of Mathematics, \\ University of Annaba, \\ P. O. Box 12, \\ Annaba 23000, \\ Algeria \\ e-mail: adjoudi@yahoo.com
}

